OPEN ACCESS

Edited by:

Carlo Garofalo,

University of Campania Luigi

Vanvitelli, Italy

Reviewed by:

Karl Martin Wissing,

University Hospital Brussels, Belgium

Kirk Campbell,

Icahn School of Medicine at Mount

Sinai, United States

*Correspondence: Domenico Santoro dsantoro@unime.it

Specialty section:

This article was submitted to

Nephrology,

a section of the journa

Frontiers in Medicine

Received: 17 May 2020 Accepted: 12 November 2020 Published: 10 December 2020

Citation

Siligato R, Gembillo G, Cernaro V,

Torre F, Salvo A, Granese R and

Santoro D (2020) Maternal and Fetal Outcomes of Pregnancy in Nephrotic

Syndrome Due to Primary

Glomerulonephritis

Front. Med. 7:563094.

doi: 10.3389/fmed.2020.563094

\section{Maternal and Fetal Outcomes of Pregnancy in Nephrotic Syndrome Due to Primary Glomerulonephritis}

\author{
Rossella Siligato ${ }^{1}$, Guido Gembillo ${ }^{2}$, Valeria Cernaro ${ }^{1}$, Francesco Torre ${ }^{1}$, Antonino Salvo ${ }^{1}$, \\ Roberta Granese ${ }^{3}$ and Domenico Santoro ${ }^{\text {* }}$ \\ ${ }^{1}$ Unit of Nephrology and Dialysis, Department of Clinical and Experimental Medicine, University of Messina, Messina, Italy, \\ ${ }^{2}$ Department of Biomedical, Dental, Morphological and Functional Imaging Sciences, University of Messina, Messina, Italy, \\ ${ }^{3}$ Unit of Gynecology, Department of Human Pathology of Adult and Childhood "G. Barresi", University of Messina, \\ Messina, Italy
}

Chronic kidney disease (CKD) affects 3\% of pregnancies, impacting on maternal and fetal outcomes, and at the same time, a recurrent question in nephrology regards gestation impact on kidney function. Observational studies stated that CKD stage, pre-existent hypertension, and proteinuria are the main predictors of possible complications, such as maternal CKD progression, maternal or fetal death, prematurity, small for gestational age (SGA) newborn, or admission to the neonatal intensive care unit. In this regard, given the prominence of proteinuria among other risk factors, we focused on primary nephrotic syndrome in pregnancy, which accounts for $0.028 \%$ of cases, and its impact on materno-fetal outcomes and kidney survival. Data extracted from literature are scattered because of the small cohorts investigated in each trial. However, they showed different outcomes for each glomerular disease, with membranous nephropathy (MN) having a better maternal and fetal prognosis than focal and segmental glomerulosclerosis (FSGS), membranoproliferative glomerulonephritis (MPGN), or minimal change disease (MCD). Nephrotic syndrome does not have to discourage women to undertake a pregnancy, but the correct management may include a specific evaluation of risk factors and follow-up for adverse materno-fetal events and/or maternal kidney disease progression.

Keywords: pregnancy, nephrotic syndrome, focal and segmental glomerulosclerosis, membranous nephropathy, membranoproliferative glomerulonephritis, minimal change disease

\section{INTRODUCTION}

In the past, fear of chronic kidney disease (CKD) progression could prevent nephrologists from sustaining their female patients' idea of conceiving or carrying on gestation, even if evidence of adverse outcomes was scattered. Today, mild CKD affects nearly $3 \%$ of women in childbearing age, whereas stage 3-5 CKD can be detected in $0.7 \%$ of same age women (1). However, the real prevalence in pregnant patients cannot be assessed, given that gestational screening guidelines usually include only urinalysis to identify urinary tract infections (UTIs) or proteinuria. On the other hand, mild CKD reports at more extended laboratory examinations performed in pregnancy are rising, likely because of increasing maternal age and higher incidence of hypertension, obesity, diabetes mellitus type 2 (DMT2), or smoking habits. Thus, first pregnancy is frequently the real touchstone to discover metabolic disorders, impaired renal function, or UTIs. Among primary kidney diseases, glomerulopathies, particularly nephrotic syndrome, are 
rare conditions whose management in pregnancy must include specific follow-up and evaluation of risk factors for adverse materno-fetal events and/or maternal renal disease progression. All the trials based on miscellaneous CKD cases demonstrate that stage of renal impairment, but above all baseline proteinuria and hypertension, are significant risk factors for adverse outcomes. The aim of our review is to investigate the outcomes of women affected by nephrotic syndrome due to primary glomerulopathies, including focal and segmental glomerulosclerosis (FSGS), minimal change disease (MCD), membranous nephropathy $(\mathrm{MN})$, and membranoproliferative glomerulonephritis (MPGN).

\section{NEPHROTIC SYNDROME AND PREGNANCY, A DIFFICULT DATA ANALYSIS}

Ethical concerns about pregnancy prevent starting interventional randomized controlled trials, so literature in the field is mainly based on retrospective or, to a lower extent, prospective observational studies. Moreover, statistical analysis of maternofetal or kidney outcomes in those trials is often based on CKD stage rather than on the specific underlying causes that range from hypertension and/or proteinuria, diabetic nephropathy (DN), and glomerulonephritis to autosomal dominant polycystic kidney disease (ADPKD), kidney malformations, nephrolithiasis, or chronic UTIs. It is worth considering that some of them may expose patients to specific risks, such as elevated rate of fetal deformity in DN or hereditary transmission of ADPKD, in addition to other adverse outcomes as maternal CKD progression or maternal and fetal death, prematurity, small for gestational age (SGA) babies, or admission to the neonatal intensive care unit (NICU), which are common for all CKD causes. Finally, long-term effects of moderate-severe CKD have to be contemplated and result in a higher incidence of hypertension and ischemic heart disease in women affected by maternofetal complications. Except for IgA nephropathy (IgAN) and systemic lupus erythematosus that result in the most common glomerular diseases in childbearing age women, investigating peculiar outcomes of other glomerulopathies appears to be quite complex because it is hard to find a pregnant cohort big enough to reach statistical significance after stratification of individual risk factors.

\section{Superimposed Preeclampsia or Kidney Disease Progression?}

Nephrotic syndrome is estimated to occur in $0.028 \%$ of gestations (2), but an increased or new-onset proteinuria has to be distinguished from preeclampsia. Usually, time and clinical presentation are diagnostic criteria when proteinuria rises twice the baseline value if it is $>2 \mathrm{~g} /$ day or 5 -fold if it is lower, after gestation week 20, associated with the onset of hypertension and severe headaches or epigastric pain, as well as with laboratory abnormalities, such as thrombocytopenia and titer of serum aspartate aminotransferase $>70 \mathrm{U} / \mathrm{L}(3)$.
At the moment, podocyturia and nephrinuria have been considered as direct urinary markers of podocyte damage. Podocyturia has higher sensitivity (88\%) and specificity (79\%) with immunofluorescence techniques targeting podocalyxin rather than synaptopodin or podocin. Nephrinuria is defined as an increase in urine nephrin level, preceding the onset of clinical manifestation of preeclampsia by about 9 days, with the best sensitivity (100\%) and specificity (97\%) during the 3rd trimester of pregnancy. However, their benefit as predictors of preeclampsia may not be extremely relevant in differential diagnosis between this condition and a new-onset or progression of nephrotic syndrome. Placental growth factor (PlGF) is an angiogenic factor whose production increases until gestation weeks 26-30 in healthy women. On the contrary, in preeclampsia, reduced titer of PlGF may be an expression of a placental dysfunction. Soluble FMS-like tyrosine kinase receptor 1 (sFlt-1) counteracts $\mathrm{PlGF}$ and also demonstrated to downregulate the expression of slit diaphragm components responsible for proteinuria. sFlt-1/PlGF ratio appears to be a promising placental specific marker of preeclampsia, in terms of both specificity and sensitivity (4), and correlates with disease severity, anticipating its onset by about 5 weeks.

\section{Specific Outcomes in Primary Nephrotic Syndrome}

Maternal and fetal outcomes in FSGS patients were studied from 1980 to 2017 in several case series of pregnant women affected by biopsy-proven primary glomerular diseases. Except for a prospective trial and two retrospective studies each including only a case of FSGS and reporting, respectively, a case of progression of kidney disease, stillbirth, and preterm delivery (5-7), other studies included a number of pregnancies ranging from 10 to 84 , with term delivery in $16-78.5 \%$ of cases $(8-14)$. The analysis of adverse materno-fetal outcomes showed a high rate of incidence of spontaneous abortion (5.9-28.6\%), preterm delivery (5.9-58.8\%), and perinatal death (11.7-45.2\%) (8-14). Regarding maternal renal outcomes, hypertension emerged in $20-71 \%$ of pregnancies, but persisted after 6 months after delivery in $4-66 \%$ of women $(8-14)$. The highest rate of postpartum hypertension was reached in a Saudi cohort of 84 pregnancies in 18 women affected by FSGS, who had undergone 38 pregnancies before the onset of nephrotic syndrome. Malik et al. compared the materno-fetal outcomes of their pregnancies demonstrating no statistical significance of the increased abortion rate registered after FSGS diagnosis. Conversely, higher rate of preterm delivery $(5.9 \%, p=0.0006)$ and SGA infants $(16.6 \%, p=0.0001)$ in FSGS cohort than in control group gestations resulted to be significant (13). Interestingly, proteinuria increased from $3.8 \pm 3.4 \mathrm{~g} /$ day in the first pregnancy to $4.8 \pm 2.0 \mathrm{~g} /$ day in the last one, but the difference was not significant $(p=0.275)$. At last follow-up, occurring at a mean of $10.5 \pm 3.3$ years after FSGS diagnosis, half of their cohort had CKD progression, and four women $(22.2 \%)$ reached end-stage renal disease (ESRD), requiring renal replacement therapy (13). These results are in line with the previous experience of Packham et al., who reported a transient impairment of renal function in $49 \%$ of pregnancies, persisting 
in four patients and leading to ESRD in three of them (19\%), respectively, after 2,7 , and 18 years, in contrast to higher renal survival rate ( $93 \%$ at 10 years from diagnosis) of a control group of 58 non-pregnant women affected by FSGS (11).

Six studies evaluated outcomes of pregnancy and MCD (2, $5,6,8,14)$. Two retrospective studies, including only seven MCD patients in their report, showed $50-66 \%$ of SGA newborns. Regarding renal outcomes, they appeared limited to gestation, with increased level of proteinuria and onset of hypertension registered in only a pregnancy for each trial, both resolving postpartum $(2,5)$. In line with these results, Surian (8) recorded no complications in two pregnancies. In 1985, Abe et al. studied 17 pregnancies of 10 MCD patients, highlighting materno-fetal complications (SGA infants, preterm delivery, polyhydramnios, premature abruptio placentae, and severe bleeding after delivery) in $12 \%$ of gestations and fetal loss and abortion in 12 and $17 \%$ of cases, respectively. The main risk factors identified were hypertension and glomerular filtration rate $<70 \mathrm{ml} / \mathrm{min}(6)$. More recently, a retrospective trial included seven pregnancies of which $71.4 \%$ hesitated in preterm delivery and $14.3 \%$ in fetal loss beyond gestation week 20 . Only a patient experienced a stable increase in proteinuria postpartum (14).

Trials investigating MN and pregnancy highlighted a reduced impact of this glomerulopathy on the incidence of abortion and stillbirths in the absence of risk factors, such as nephrotic range proteinuria during pregnancy $(2,5,6,8,9,12,14-20)$. A transient development of hypertension was registered in 7.7$45.4 \%$ of pregnancies, and increased proteinuria in up to $60 \%$ of cases confirmed an association of $\mathrm{MN}$ with worse fetal and maternal outcomes. Packham et al. reported the highest rate of materno-fetal complications with fetal loss in $24 \%$ of cases, preterm delivery in $43 \%$ of pregnancies, CKD progression in $9 \%$, but the onset of hypertension in $46 \%$ of pregnant patients, and higher proteinuria in 54.5\% (17). A Saudi cohort study comparing the maternal outcomes of nine women experiencing pregnancies before $(n=21)$ and after $(n=30)$ MN diagnosis demonstrated that the different rates of abortion ( 4.7 vs. $3.3 \%$, $p=0.843$ ), preterm delivery ( 0 vs. $6.6 \%, p=0.152$ ), and perinatal mortality ( 0 vs. $3.3 \%, p=0.317$ ) between the two groups were not statistically significant (18). Moreover, the rise in mean serum creatinine level experienced from the first gestation after MN diagnosis to the last one of the same cohort was also not significant (mean SCr $68 \pm 17$ vs. $80 \pm 48 \mu \mathrm{mol} / \mathrm{L}$, respectively, $p=0.489)$ (18).

MPGN defines a pathologic pattern characterized by subendothelial, subepithelial, and mesangial deposits of immune complexes (due to infections, autoimmune or monoclonal disorders) or of complement (due to alternative pathway dysregulation). Idiopathic MPGN cases were studied in five trials accounting for a total number of 36 patients (5-9). Two studies included only a patient and obtained opposite results, with a neonatal death (6) and a physiologic pregnancy (7). The other retrospective trials showed full-term deliveries in up to $50 \%$ of patients, whereas $16.7-23.8 \%$ had preterm delivery. Fetal death was more frequent in FSGS and MN patients evaluated in the same studies, with both an abortion rate and peripartum mortality in $0-11.1 \%$ of gestations (5-9). Moreover, Barcelò et al., stratified MPGN, FSGS, and MN patients' outcomes based on proteinuria and detected a significant association only of proteinuria levels $>2.5 \mathrm{~g} /$ day with preterm delivery and low birth weight $(1,930 \pm 301 \mathrm{~g})$ (9).

New data come from De Castro et al.'s retrospective study on 26 pregnancies in 19 patients affected by biopsy-proven primary nephrotic syndrome with a mean proteinuria of $8.33 \pm 6.7$ g/day (19). In 26 pregnancies described, glomerulopathies most represented were FSGS (42\%), MN (16\%), and IgAN (16\%), followed by MCD, MPGN, C3 glomerulonephritis (C3GN), and fibrillary glomerulonephritis, each accounting for 5\%. A new diagnosis was performed in 12 women during pregnancy at mean gestational week $18.6 \pm 9.1$, and kidney biopsy was performed in eight patients within a mean gestational age of 21 weeks. Only two minor biopsy complications were reported in this group of women (hematoma and hematuria), compared with one adverse event recorded in 11 patients who had pathologic diagnosis before or after their pregnancies (1 small arteriovenous fistula), but the procedure proved to be worth the risk because it allowed to change the management of nephrotic syndrome in six patients who started prednisone in a dose ranging from 60 to $120 \mathrm{mg} /$ day. One patient affected by known C3GN was given eculizumab. The main adverse maternal outcomes described were preeclampsia (27\%), acute kidney injury (AKI, $23 \%)$, and cellulitis (12\%), which was not associated with steroid therapy. FSGS accounted for half of AKI episodes, one of which progressed to ESRD, whereas the remaining happened in IgAN and C3GN patients. Fetal outcomes included preterm delivery and low birth weight (under 2,500 g) in 54\% of pregnancies, intrauterine growth restriction (IUGR) in $11 \%$, and NICU admission in $31 \%$. Two cases of premature rupture of membranes were identified in patients treated with high dose glucocorticoids. In this cohort, MN confirmed having the best maternal-fetal and kidney survival outcomes, with three patients with stable serum creatinine during pregnancies and a transient increase in proteinuria, which reverted to non-nephrotic range in a year after delivery. Statistical analysis of risk factors of the whole cohort confirmed the strong association of proteinuria with adverse outcomes, such as preeclampsia $(p=0.002)$, low birth weight $(p=0.002)$, and preterm delivery $(p=0.02)(19)$.

The most recent retrospective study performed by Liu et al. reported preserved kidney function but the occurrence of adverse materno-fetal complications including fetal loss (11\%), preterm delivery $(26 \%)$, and severe preeclampsia (15\%) in 27 pregnancies from 2008 to 2018 in a cohort of women affected by MN (20). Consistent with De Castro' observations (19), Liu also demonstrated a correlation of proteinuria before week 20 (intended as both time-averaged urine protein, OR 67.5, $p<$ 0.001 ; maximum proteinuria $>3.5 \mathrm{~g} /$ day, OR $29.3, p=0.001$ ), hypoalbuminemia (time-averaged albumin, OR 67.5, $p<0.001$; minimum serum albumin $<25 \mathrm{~g} / \mathrm{L}$, OR $10.9, p=0.01$ ), and no-remission during pregnancy (OR 21.6, $p=0.004$ ) with adverse materno-fetal outcomes. In particular, time-averaged proteinuria and serum albumin were associated with birth weight percentile of neonates. Moreover, anti-PLA2R antibody positivity $(p=0.03)$ appeared as a specific risk factor for pregnant $\mathrm{MN}$ patients (20). Interestingly, a case report of PLA2R positive $M N$ 
TABLE 1 | Retrospective trials on primary nephrotic syndrome and materno-fetal and renal outcomes.

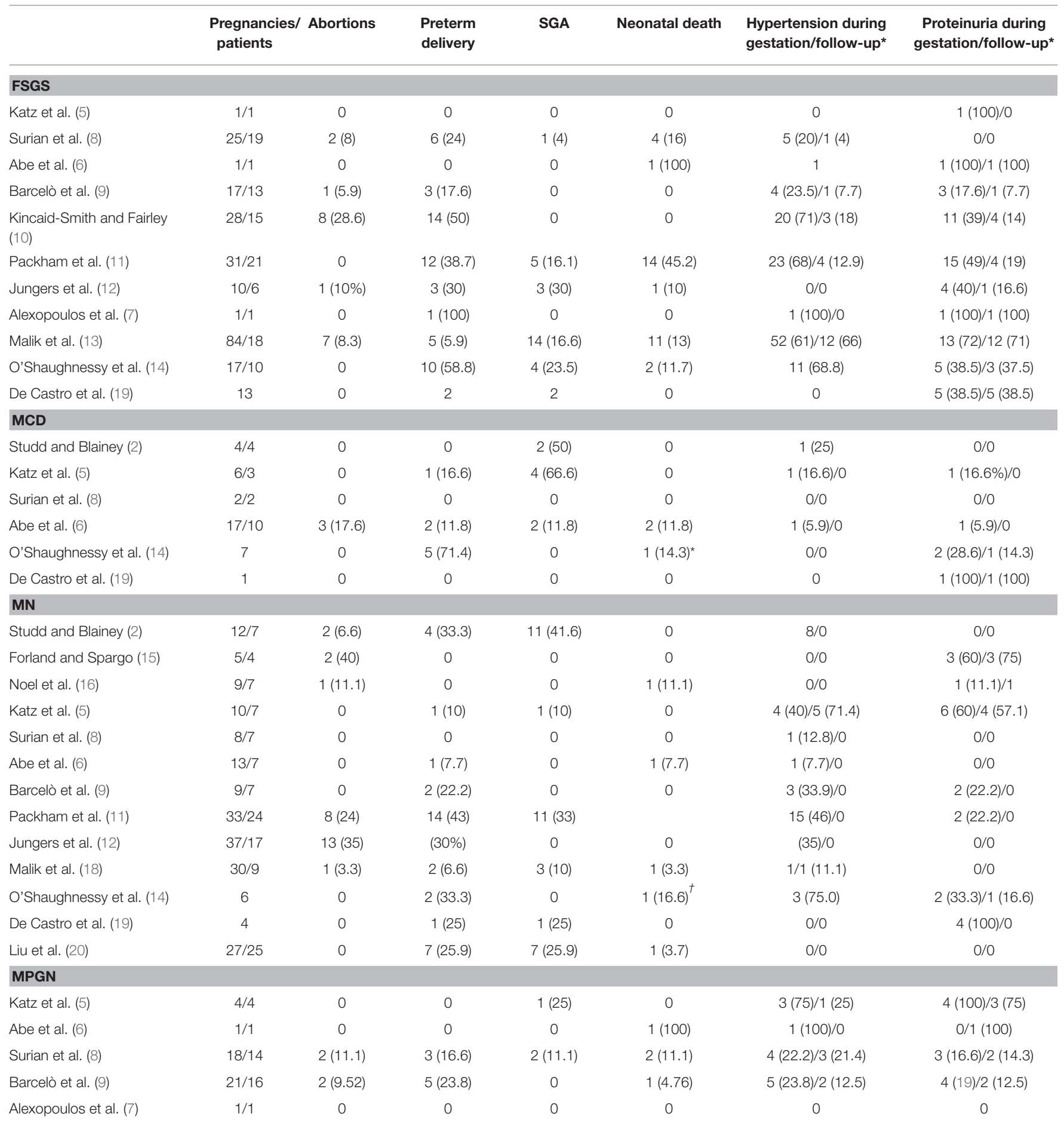

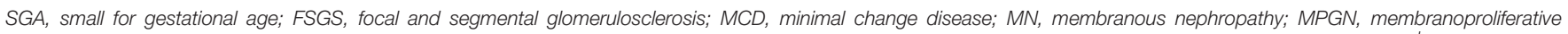

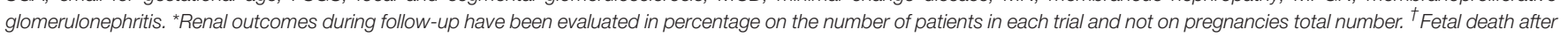
20 weeks of gestation. All values represent $n$ (\%).

patient undergoing pregnancy demonstrated the transplacental passage of these autoantibodies, which were detected in the fetus' cord blood (21). Sachdeva et al. confirmed this finding in a new case report and also showed a PLA2R passage to newborn during breastfeeding, which raises questions about the opportunity of this practice and of checking PLA2R titer, serum albumin, and urinalysis in children of mother affected by primary MN (22). 


\section{Symptomatic Treatment of Nephrotic Syndrome in Pregnancy}

Primary glomerulonephritis may require the administration of immunosuppressive drugs, such as mycophenolic acid and cyclophosphamide, whose teratogen risk is known and should therefore be replaced before gestation (23). Monoclonal antibodies, such as anti-CD20 rituximab, should be avoided because of their transplacental transport in the last trimesters of pregnancy, which can induce transient B cell depletion in fetuses (24). Conversely, short half-life corticosteroids, such as prednisone and methylprednisolone, are considered a valid alternative because of their inactivation by placental $11 \beta$ hydroxysteroid dehydrogenase type 2 that prevents steroid adverse effects on fetus (23). Calcineurin inhibitors (CNIs), such as cyclosporin (CsA) or tacrolimus (TAC), appear to be safe, with no evidence of increased risk of teratogenicity (25), and experimental rat models show how CsA transplacental passage is impaired by P-glycoprotein (26).

Angiotensin-converting enzyme inhibitors (ACEIs) and/or angiotensin II receptor blockers (ARBs) are widely recommended in KDIGO guidelines as the first-line therapy of nephrotic syndrome for their nephroprotective effects (27). Their teratogen effect on the cardiovascular, urinary, skeletal, and central nervous systems makes them contraindicated in pregnancy particularly during the second trimester of gestation, so general advice is to have them immediately discontinued at the beginning of pregnancy (28-30). Hoeltzenbein et al. conducted a prospective observational trial comparing 329 women accidentally exposed to ACEIs during their first trimester of gestation and no longer than week 20 and 654 control pregnant women without a history of hypertension (31). The significantly increased rate of major birth defects in the ACEIs cohort confirmed the results of previous studies on these drugs in pregnancy $(32,33)$, but they also investigated maternal hypertension impact on malformations, already demonstrated in other trials $(34,35)$. Comparing patients affected by chronic hypertension exposed to ACEIs vs. patients treated with methyldopa, they concluded that malformations rate was not significantly different, also suggesting the potential role of severe hypertension per se as a risk factor for adverse fetal outcomes (31).

A safe option to maintain blood pressure in pregnant women affected by CKD within values of $135 / 85 \mathrm{mmHg}$ is represented by $\alpha 2$ adrenergic receptor blocker, such as methyldopa, whereas clonidine may impact fetal growth, and it is usually avoided for high risk of hypertensive rebound at discontinuation. Other antihypertensive choices are represented by calcium blockers, such as long-acting nifedipine or labetalol, a combined $\alpha$ - and $\beta$-blocker, with efficacy and safety comparable to methyldopa. Other $\beta$-blockers represent a second choice because of the risk of adverse events in newborns (usually transient bradycardia, hypotension, and hypoglycemia). Diuretics are usually avoided in pregnancy and may be adopted in nephrotic syndrome refractory to immunosuppressive therapy under strict control to avoid volume depletion and AKI risk.

Regarding albumin infusion in nephrotic syndrome in pregnancy, no study is conclusive about its potential benefit.
On the contrary, hyperfiltration risk has to be considered, with a paradox increase in proteinuria, and this discourages the systematic use of albumin in this setting $(36,37)$.

Nephrotic syndrome is also characterized by a hypercoagulability state that has to be treated when the albumin level is lower than $2 \mathrm{~g} / \mathrm{dl}$. Low molecular-weight heparin is a valid choice for both prophylaxis and treatment of thromboembolic complications during pregnancy and postpartum, because it does not cross the placenta. Heparin should be discontinued before labor induction or planned cesarean section, but can be resumed after delivery (38). During pregnancy, low-dose acetylsalicylate is also a useful option preventing preeclampsia in high-risk patients, such as CKD and proteinuric patients. Despite guidelines suggesting to start therapy after the first trimester to avoid hemorrhagic risk in case of spontaneous abortions, the protective effects on placentation are higher when acetylsalicylate is started early $(39,40)$.

The positive effect of a low protein diet with supplementation of amino acids and ketoacids in pregnant CKD patients has to be considered as a therapeutic option, in the absence of a specific anti-proteinuric therapy $(41,42)$.

\section{CONCLUSIONS}

Our review focused on materno-fetal and kidney survival outcomes reported in pregnant patients with nephrotic syndrome (Table 1). Baseline levels of proteinuria, as well as hypertension and CKD stage, are the most important risk factors for poor outcomes, as confirmed in other trials involving pregnant women affected by CKD. Incidence of C-sections and preterm deliveries are higher than healthy controls, as reported by Piccoli et al.'s findings in a prospective trial in CKD patients (54.8 and 33.4\%, respectively) (43). Among nephrotic syndromes, MN resulted to be the most favorable in terms of outcomes, whereas MPGN and FSGS expose patients to a higher degree of both materno-fetal and renal adverse events. Adequate counseling appears to be indispensable to increase women awareness of the benefit of pregnancy planning to manage potential teratogen therapies and to minimize risks of gestational complications and disease progression, especially if a complete and stable remission is not obtained before conceiving. More prospective trials are required to better evaluate specific predictors of severe adverse outcomes and to reach statistical significance after stratifying individual risk factors.

\section{AUTHOR CONTRIBUTIONS}

RS, GG, and DS: conceptualization and writing-original draft preparation. RS, VC, GG, and DS: methodology. RS and DS: investigation. RG and DS: resources. RS, RG, and VC: data curation. RS, RG, AS, FT, VC, and DS: writing-review and editing. RS, RG, AS, FT, and DS: visualization. RG, DS, and GG: supervision. All authors contributed to the article and approved the submitted version. 


\section{REFERENCES}

1. Williams D, Davidson J. Chronic kidney disease in pregnancy. BMJ. (2008) 336:211-5. doi: 10.1136/bmj.39406.652986.BE

2. Studd JW, Blainey JD. Pregnancy and the nephrotic syndrome. BMJ. (1969) 1:276-80. doi: 10.1136/bmj.1.5639.276

3. American College of Obstetricians and Gynecologists, Task force on hypertension in pregnancy. Hypertension in pregnancy. Report of the American College of Obstetricians and Gynecologists' Task Force on Hypertension in Pregnancy. Obstet Gynecol. (2013) 122:1122-31. doi: 10.1097/01.AOG.0000437382.03963.88

4. Kerley RN, McCarthy C. Biomarkers of glomerular dysfunction in pre-eclampsia - A systematic review. Pregn Hypertens. (2018) 14:26572. doi: 10.1016/j.preghy.2018.03.002

5. Katz AI, Davison JM, Hayslett JP, Singson E, Lindheimer MD. Pregnancy in women with kidney disease. Kidney int. (1980) 18:192--206. doi: 10.1038/ki.1980.128

6. Abe S, Amagasaki Y, Konishi K, Kato E, Sakaguchi H, Iyori S. The influence of antecedent renal disease on pregnancy. Am J Obstet Gynecol. (1985) 153:508-14. doi: 10.1016/0002-9378(85)90463-6

7. Alexopoulos E, Bili H, Tampakoudis P, Economidou D, Sakellariou G, Mantalenakis S, et al. Outcome of pregnancy in women with glomerular diseases. Renal Fail. (1996) 19:121-9. doi: 10.3109/08860229609052782

8. Surian M, Imbasciati E, Cosci P, Banfi G, Barbiano di Belgiojoso G, Brancaccio D, et al. Glomerular disease and pregnancy. A study of 123 pregnancies in patients with primary and secondary glomerular diseases. Nephron. (1984) 36:101-5. doi: 10.1159/000183126

9. Barcelò P, Lòpez-Lillo J, Cabero L, Del Rìo G. Successful pregnancy in primary glomerular disease. Kidney Int. (1986) 30:914-9. doi: 10.1038/ki.1986.272

10. Kincaid-Smith P, Fairley KF. Renal disease in pregnancy. Three controversial areas: Mesangial IgA nephropathy, focal glomerular sclerosis (focal and segmental hyalinosis and sclerosis), and reflux nephropathy. Am J Kidney Dis. (1987) 9:328-33. doi: 10.1016/S0272-6386(87)80131-2

11. Packham DK, North RA, Fairley KF, Ihle BU, Whitworth JA, Kincaid-Smith P. Pregnancy in women with primary focal and segmental hyalinosis and sclerosis. Clin Nephrol. (1988) 29:185-92.

12. Jungers P, Houillier P, Forget D, Henry-Amar M. Specific controversies concerning the natural history of renal disease in pregnancy. Am J Kidney Dis. (1991) 17:116-22. doi: 10.1016/S0272-6386(12)81114-0

13. Malik GH, Al-Mohaya S, Al-Wakeel J, Shaikh JF, Al-Hozaim W, Al-Duhaimi $\mathrm{H}$, et al. Repeated pregnancies in patients with primary focal segmental glomerulosclerosis. Indian J Nephrol. (2002) 12:33-9.

14. O'Shaughnessy MM, Jobson MA, Sims K, Liberty AL, Nachman PH, Pendergraft WF. Pregnancy outcomes in patients with glomerular disease attending a single academic center in North Carolina. Am J Nephrol. (2017) 45:442-51. doi: 10.1159/000471894

15. Forland M, Spargo BH. Clinicopathological correlations in idiopathic nephrotic syndrome with membranous nephropathy. Nephron. (1969) 6:498525. doi: $10.1159 / 000179748$

16. Noel LH, Zanetti M, Droz D, Barbanel C. Long-term prognosis of idiopathic membranous glomerulonephritis. Am J Med. (1979) 66:8290. doi: 10.1016/0002-9343(79)90486-8

17. Packham DK, North RA, Fairley KF, Whitworth JA, Kincaid-Smith P. Membranous glomerulonephritis and pregnancy. Clin Nephrol. (1987) 28:5664.

18. Malik GH, Al-Harbi AS, Al-Mohaya S, Al-Wakeel J, Al-Hozaim W, Kechrid M, et al. Repeated pregnancies in patients with primary membranous glomerulonephritis. Nephron. (2002) 91:21-4. doi: 10.1159/0000 57600

19. De Castro I, Easterling TR, Bansal N, Jefferson JA. Nephrotic syndrome in pregnancy poses risks with both maternal and fetal complications. Kidney Int. (2017) 91:1464-72. doi: 10.1016/j.kint.2016.12.019

20. Liu Z, Cui Z, He Y, Zhang Y, Wang F, Wang X, et al. Membranous nephropathy in pregnancy. Am J Nephrol. (2020) 51:304-17. doi: 10.1159/000505175

21. Al-Rabadi L, Ayalon R, Bonegio RG, Ballard JE, Fujii AM, Henderson JM, et al. Pregnancy in a patient with primary membranous nephropathy and circulating anti-PLA2R antibodies: a case report. Am J Kidney Dis. (2016) 67:775-8. doi: 10.1053/j.ajkd.2015.10.031
22. Sachdeva M, Beck LH, Jr, Miller I, Bijol V, Fishbane S. Phospholipase $\mathrm{A}_{2}$ receptor antibody-positive pregnancy: a case report. Am J Kidney Dis. (2020) 76:586-9. doi: 10.1053/j.ajkd.2019.11.011

23. Ponticelli C, Moroni G. Fetal toxicity of immunosuppressive drugs in pregnancy. J Clin Med. (2018) 7:552. doi: 10.3390/jcm7120552

24. Chakravarty EF, Murray ER, Kelman A, Farmer P. Pregnancy outcomes after maternal exposure to rituximab. Blood. (2011) 117:1499-506. doi: 10.1182/blood-2010-07-295444

25. Shah S, Venkatesan RL, Gupta A, Sanghavi MK, Welge J, Johansen R, et al. Pregnancy outcomes in women with kidney transplant: metaanalysis and systematic review. BMC Nephrol. (2019) 20:24. doi: 10.1186/s12882-019-1213-5

26. Pávek P, Fendrich Z, Staud F, Malákova J, Brozmanová H, Láznícek M, et al. Influence of P-glycoprotein on the transplacental passage of cyclosporine. $J$ Pharm Sci. (2001) 90:1583-92. doi: 10.1002/jps.1108

27. Kidney Disease: Improving Global Outcomes (KDIGO) Glomerulonephritis Work Group. KDIGO Clinical Practice Guideline for Glomerulonephritis. Kidney Int Suppl. (2012) 2:139-274. doi: 10.1038/kisup.2012.9

28. Koren G. Hypertension: ACE inhibitor use in pregnancy- setting the record straight. Nat Rev Cardiol. (2011) 9:7-8. doi: 10.1038/nrcardio.2011.179

29. Lewis G, Maxwell AP. Should women with diabetic nephropathy considering pregnancy continue ACE inhibitor or angiotensin II receptor blocker therapy until pregnancy is confirmed? Diabetologia. (2014) 57:10823. doi: 10.1007/s00125-014-3188-x

30. Cooper WO, Hernandez-Diaz S, Arbogast PG, Dudley JA, Dyer S, Gideon PS, et al. Major congenital malformations after first-trimester exposure to ACE inhibitors. N Engl J Med. (2006) 354:2443-51 doi: 10.1056/NEJMoa055202

31. Hoeltzenbein M, Tissen-Diabaté T, Fietz, A.-K, Zinke S, Kayser $A$, et al. Increased rate of birth defects after first trimester use of angiotensin converting enzyme inhibitors - Treatment or hypertension related? An observational cohort study. Pregn Hypertens. (2018) 13:65-71. doi: 10.1016/j.preghy.2018.04.022

32. Bateman BT, Patorno E, Desai RJ, Seely EW, Mogun H, Dejene SZ, et al. Angiotensin converting enzyme inhibitors and the risk of congenital malformations. Obstet Gynecol. (2017) 129:174-84. doi: 10.1097/AOG.0000000000001775

33. Li DK, Yang C, Andrade S, Tavares V, Ferber JR. Maternal exposure to angiotensin converting enzyme inhibitors in the first trimester and risk of malformations in offspring: a retrospective cohort study. BMJ. (2011) 343:d5931. doi: 10.1136/bmj.d5931

34. Bateman BT, Huybrechts KF, Fischer MA, Seely EW, Ecker JL, Oberg AS, et al. Chronic hypertension in pregnancy and the risk of congenital malformations: a cohort study. Am J Obstet Gynecol. (2015) 212:337 e114. doi: 10.1016/j.ajog.2014.09.031

35. Ramakrishnan A, Lee LJ, Mitchell LE, Agopian AJ. Maternal hypertension during pregnancy and the risk of congenital heart defects in offspring: a systematic review and meta-analysis. Pediatr Cardiol. (2015) 36:144251. doi: 10.1007/s00246-015-1182-9

36. Boldt J. Use of albumin: an update. Br J Anaesth. (2010) 104:27684. doi: 10.1093/bja/aep393

37. Cabiddu G, Castellino S, Gernone G, Santoro D, Moroni G, Giannattasio $\mathrm{M}$, et al. A best practice position statement on pregnancy in chronic kidney disease: the Italian Study Group on Kidney and Pregnancy. J Nephrol. (2016) 29:277-303. doi: 10.1007/s40620-016-0285-6

38. Grandone E, Villani M, Tiscia GL. Aspirin and heparin in pregnancy. Exp Opin Pharmacother. (2015) 16:1793803. doi: 10.1517/14656566.2015.1066335

39. Henderson JT, Whitlock EP, O'Conner E, Senger CA, Thompson JH, Rowland MG. Low-Dose Aspirin for the Prevention of Morbidity and Mortality From Preeclampsia: A Systematic Evidence Review for the U.S. Preventive Services Task Force. Rockville, MD: Agency for Healthcare Research and Quality (US). Report No.: 14-05207-EF-1 (2014).

40. Roberge S, Villa P, Nicolaides K, Giguère Y, Vainio M, Bakthi A, et al. Early administration of low-dose aspirin for the prevention of preterm and term preeclampsia: a systematic review and meta-analysis. Fetal Diagn Ther. (2012) 31:141-6. doi: 10.1159/000336662

41. Attini R, Leone F, Montersino B, Fassio F, Minelli F, Colla L, et al Pregnancy, proteinuria, plant-based supplemented diets and focal 
segmental glomerulosclerosis: a report on three cases and critical appraisal of the literature. Nutrients. (2017) 9:E770. doi: 10.3390/nu90 70770

42. Attini R, Montersino B, Leone F, Minelli F, Fassio F, Rossetti MM, et al. Dialysis or a plant-based diet in advanced CKD in pregnancy? A case report and critical appraisal of the literature. J Clin Med. (2019) 8:123. doi: 10.3390/jcm80 10123

43. Piccoli GB, Cabiddu G, Attini R, Vigotti FN, Maxia S, Lepori N, et al. Risk of adverse pregnancy outcomes in women with CKD. J Am Soc Nephrol. (2015) 26:2011-22. doi: 10.1681/ASN.20140 50459
Conflict of Interest: The authors declare that the research was conducted in the absence of any commercial or financial relationships that could be construed as a potential conflict of interest.

Copyright $\odot 2020$ Siligato, Gembillo, Cernaro, Torre, Salvo, Granese and Santoro. This is an open-access article distributed under the terms of the Creative Commons Attribution License (CC BY). The use, distribution or reproduction in other forums is permitted, provided the original author(s) and the copyright owner(s) are credited and that the original publication in this journal is cited, in accordance with accepted academic practice. No use, distribution or reproduction is permitted which does not comply with these terms. 